\title{
Management of subtrochanteric femur fractures: is open reduction associated with poor outcomes?
}

\author{
Michalis Panteli ${ }^{1,2,3}$ (1) James Shen Hwa Vun ${ }^{1,2,3} \cdot$ Robert Michael West $^{4} \cdot$ Anthony John Howard $^{1,2,3}$. \\ Ippokratis Pountos $^{1,2} \cdot$ Peter Vasilios Giannoudis ${ }^{1,2,3,5}$
}

Received: 27 August 2021 / Accepted: 8 November 2021 / Published online: 26 November 2021

(c) The Author(s) 2021

\begin{abstract}
Purpose The aim of this study was to identify factors associated with the need for open reduction in subtrochanteric femoral fractures and investigate the effect of cerclage wiring compared to open reduction alone, on the development of complications, especially infection and non-union.

Methods All consecutive patients with a fracture involving the subtrochanteric region were retrospectively identified, over an 8-year period. Data documented and analysed included patient demographics, fracture characteristics, patient comorbidities, time to fracture union and development of complications.

Results A total of 512 patients met the inclusion criteria (523 fractures). Open reduction was performed in 48\% (247) of the fractures. Following matching and regression analysis, we identified diaphyseal extension of the fracture to be associated with an open reduction (OR: $2.30 ; 95 \%$ CI $1.45-3.65 ; p<0.001)$. Open reduction was also associated with an increased risk of superficial infection (OR: 7.88 ; 95\% CI 1.63-38.16; $p=0.010$ ), transfusion within $48 \mathrm{~h}$ following surgery (OR: $2.44 ; 95 \%$ CI $1.96-4.87 ; p<0.001$ ) and a prolonged surgical time (OR: 3.09; 95\% CI 1.96-4.87; $p<0.001$ ). The risk of non-union, deep infection and overall mortality was not increased with open reduction. The use of cerclage wires [50 out of 201 fractures (24.9\%) treated with an open reduction] to achieve anatomical reduction as compared to open reduction alone significantly reduced the risk of non-union (OR: $0.20 ; 95 \%$ CI $0.06-0.74 ; p=0.015)$.

Conclusion Open reduction of subtrochanteric fractures is not associated with an increased risk of deep infection and nonunion, even though it is associated with an increased risk of superficial infection, prolonged surgical time and transfusion. The use of cerclage wire is associated with reduced risk of non-union with little evidence of an increase in complications. Level of evidence III.
\end{abstract}

Keywords Subtrochanteric $\cdot$ Open reduction $\cdot$ Cerclage wiring $\cdot$ Complications $\cdot$ Infection $\cdot$ Non-union

Michalis Panteli

michalispanteli@gmail.com

James Shen Hwa Vun

j.vun@nhs.net

Robert Michael West

R.M.West@leeds.ac.uk

Anthony John Howard anthonyjhoward@aol.com

Ippokratis Pountos

hippoun@gmail.com

Peter Vasilios Giannoudis pgiannoudi@aol.com
1 Academic Department of Trauma and Orthopaedics, School of Medicine, University of Leeds, Clarendon Wing, Level D, Great George Street, Leeds LS1 3EX, West Yorkshire, UK

2 Leeds Institute of Rheumatic and Musculoskeletal Medicine, University of Leeds, Leeds, UK

3 Leeds Orthopaedic \& Trauma Sciences, Leeds General Infirmary, University of Leeds, Leeds, UK

4 Leeds Institute of Health Sciences, University of Leeds, Leeds, UK

5 NIHR Leeds Biomedical Research Unit, Chapel Allerton Hospital, Leeds, UK 


\section{Introduction}

Subtrochanteric fractures are defined as those encountered between the inferior border of lesser trochanter and $5 \mathrm{~cm}$ distal to it [1]. They generally account for $5-34 \%$ of all proximal femoral fractures [2-4], and the 'gold standard' of their treatment is intramedullary (IM) nailing. Their management can be very challenging because of their unique biomechanical and anatomical features (moderate vascularity and high concentration of biomechanical stresses) [5-9]. Furthermore, the multiple deforming forces acting on the fracture fragments make their reduction difficult. Consequently, it is not surprising that a $4.7 \%$ reoperation rate has been reported related to fracture non-union and metalwork failure $[3,10]$.

When closed reduction fails to improve fracture alignment, open reduction has been recommended [11-13]. However, open reduction has been associated with such complications as infection, non-union, prolonged surgical time and blood loss requiring blood transfusion [11-13]. Interestingly, published evidence remains conflicting, with some studies reporting an increased incidence of non-union and infection $[14,15]$, whilst other studies report no difference with regard to non-union, infection, transfusion rates and fracture vascularity $[12,13]$. Overall, there appears to be lack of strong evidence comparing the outcomes of open versus closed reduction in the management of subtrochanteric femoral fractures treated with cephalomedullary nailing.

Therefore, the aim of our study was to report on the factors associated with the need for open reduction and to investigate whether open reduction is associated with a higher risk for complications. Moreover, to assess if cerclage wiring has an impact on the development of fracture non-union. Our hypothesis was that open reduction is associated with an increased risk of infection and non-union, whilst the use of cerclage wires further increases this risk.

\section{Methods}

Following institutional board approval (LTH\#2591), we conducted a retrospective analysis of all consecutive eligible patients presenting in a Level I Trauma Centre over a period of eight years (2009-2016). We included all skeletally mature patients presenting with a fracture extending through the subtrochanteric region that was subsequently treated with an IM nail. Exclusion criteria included the following: pathological fractures (secondary to malignancy or atypical fractures), patients receiving prophylactic nailing for tumours (without a fracture) or incomplete fractures and patients who had their primary operation in other institutions.
Information collected included demographics and comorbidities, operation details, complications and outcomes. Fractures were classified according to the Russell Taylor classification system $[16,17]$. Radiographic features were also analysed, with all radiographic measurements being performed independently by MP and JV, with any disagreements resolved by the senior author (PVG).

All patients were managed by the multidisciplinary team, under a set protocol. All patients were positioned supine on a traction table, with post in the perineal area and with the contralateral leg held into lithotomy position. Closed reduction was first attempted and if unsuccessful, open reduction was performed. The use of clamp assisted reduction and cerclage wires was left to the discretion of the operating surgeon. Post-operatively, patients followed a strict physiotherapy regime aiming for early mobilisation and were closely monitored for complications. Superficial infection was defined as erythema, pain, swelling, discharge and delayed wound healing, along with raised inflammatory markers in the early post-operative period [18]. Deep infection was defined as infection involving the fascial, muscular layers and beyond, surrounding the metalwork and necessitating further surgical interventions [19]. Superficial infections were generally treated with oral antibiotics, as compared to deep infections that were treated with a combination of intravenous (IV) antibiotics, wound debridement and removal/ revision of implants, as deemed appropriate.

\section{Statistical analysis}

Statistical analysis was performed using the computing environment $R$ ( $R$ version 3.6.0) [20]. Basic demographic data were presented as count (percentage) or as mean \pm SD. Parametric data were analysed using a Welch unpaired independent t-test, whilst count data were analysed using a Pearson's chi square test. A $p$ value of $<0.05$ was considered as significant. To reduce the risk of bias from patient or injury characteristics, the groups were randomly matched \{smatch function provided by Lewer D [21]\} by terms of age ( \pm 5 years), gender and mechanism of injury (low or high energy). The matching ratio was 1 and matching was repeated using the first five seeding combinations to ensure no significant difference was observed in the outcomes of each matching combination. A simple logistic regression model was used for the initial analysis, to identify potential unadjusted associations with open reduction. Then by removing covariates in a stepwise fashion according to their likelihood-ratio chi-square $p$ value, we developed a revised adjusted model of multiple logistic regression to investigate associations with open reduction. The same methods were used to investigate associations with cerclage wiring. 


\section{Statistical power}

When occurrence of characteristics is close to $50 \%$, the study can detect with $80 \%$ power $(\alpha=0.05)$ a difference in prevalence of $12.1 \%$, whereas when occurrence of characteristics is close to $10 \%$, the study can detect with $80 \%$ power $(\alpha=0.05)$ a difference in prevalence of $8.8 \%$. We, therefore, look for differences in the occurrence of characteristics which are more than $10 \%$.

\section{Results}

\section{Demographics, mechanism of injury and operative characteristics}

A total of 512 patients (523 fractures; 205 males) fulfilled the inclusion criteria. There was a bimodal male, unimodal older female age distribution, whilst the commonest mechanism of injury was low energy injury.

Overall, $48 \%$ of subtrochanteric fractures (247 fractures) required an open reduction to achieve satisfactory reduction and fixation. Matching was then performed to account for the effect of age, gender and type of injury. The mean age of the matched open reduction group was $75.59 \pm 17.93$ years and $75.19 \pm 17.70$ years in the closed reduction group (Table 1).

In the open reduction group, the time to operation was $2.14 \pm 2.77$ days, duration of operation was $125.22 \pm 47.36 \mathrm{~min}$, with a consultant as senior surgeon in 95 cases (47.7\%), a length of hospital stay (LOS) of $24.42 \pm 18.70$ days and reduction angle was neutral $\left(5^{\circ}\right.$ valgus to $5^{\circ}$ varus) in $70.5 \%$ of patients. Within the closed reduction group, the time to operation was $1.83 \pm 1.76$ days, duration of operation was $96.58 \pm 35.60 \mathrm{~min}$, a consultant as senior surgeon in 77 cases $(38.5 \%)$, LOS was $23.01 \pm 19.80$ days and reduction angle was neutral $\left(5^{\circ}\right.$ valgus to $5^{\circ}$ varus) in $74.9 \%$ of patients.

When comparing the demographic factors, injury characteristics, comorbidities and operative characteristics of subtrochanteric fractures requiring open reduction against those having a closed reduction, duration of operation [OR: 3.31 $(2.15-5.10) ; p<0.001]$ and distal fracture extension to diaphysis [OR: $2.39(1.56-3.66) ; p<0.001]$ were statistically different between the two groups (Table 2). Although there was some variation between the open and closed reduction group in terms of Russell-Taylor classification $(p=0.146)$, other than distal fracture extension to diaphysis, we failed to identify any specific fracture patterns associated with a higher risk of an open reduction. No difference in adequacy of reduction was evident comparing the two groups, as demonstrated by the gap size at the fracture site and the reduction angle.

\section{Complications}

Of all complications, only superficial infection, post-operative transfusion and nail-related complications were found to be statistically different between the two groups. Examining the deep infections (including unmatched cases), three were reported in the closed reduction group (two were reoperated) and ten in the open reduction group (eight were re-operated) (Table 3). Nail-related complications $\{24.9 \%$ [open reduction] Vs. $15.4 \%$ [closed reduction]; OR: 1.82 (1.10-2.99); $p=0.019\}$ and need for transfusion within $48 \mathrm{~h}$ post-operatively $\{64.5 \%$ [open reduction] Vs. $41.5 \%$ [closed reduction]; OR: $2.56(1.71-3.84) ; p<0.001\}$ likewise were higher in the open reduction group. Requirement for preoperative transfusion, nail failure (breakage at lag screw junction or self-dynamisation), cut-out, fracture non-union, peri-implant fractures, admission to high dependency/intensive care (HDU/ICU) unit, length of hospital stay, 30-day and 1-year mortality rate were similar between the two groups (Table 1). Regarding re-operation risk, we failed to identify any significant difference in re-operation for nail failure $(p=0.331)$ and re-operation for any cause $(p=0.410)$.

\section{Factors associated with open reduction and risk of complications}

Having adjusted for the different variables associated with open reduction, our logistic regression analysis of the matched groups revealed that open reduction was strongly associated with the following: an increased risk of superficial infection (OR: 7.88; 95\% CI 1.63-38.16; $p=0.010$ ), a distal extension of the fracture line to the diaphysis (OR: 2.30; 95\% CI 1.45-3.65; $p<0.001$ ), an increased risk of post-operative transfusion within the first $48 \mathrm{~h}$ following surgery (OR: $2.44 ; 95 \%$ CI 1.58-3.76; $p<0.001$ ) and a surgical time of greater than 120 min (OR: 3.09; 95\% CI 1.96-4.87; $p<0.001$ ) (Table 4). Deep wound infection was not significant after correction for covariates $(p=0.160)$.

\section{Subgroup analysis: 'clamp assisted only' vs. 'cerclage wiring' open reduction}

A further subgroup regression analysis (Table 5) of all matched fractures requiring open reduction (matched against age, gender and mechanism of injury) revealed that open reduction using cerclage wiring was more commonly necessitated when there was a distal fracture extension (OR: 10.23 ; 95\% CI 4.81-21.78; $p<0.001$ ). Although open reduction with cerclage wiring was associated with a prolonged surgical time (OR 6.43; 95\% CI 3.12-13.26; $p<0.001$ ), it was associated with a smaller risk of developing a non-union when compared to 'clamp assisted only' open reduction (OR: 0.20 ; 95\% CI 0.06-0.74; $p=0.015$ ). 
Table 1 Table presenting the demographics/characteristics of patients having a subtrochanteric fracture treated with a long cephalomedullary nail, stratified according to open reduction

\begin{tabular}{|c|c|c|c|}
\hline Demographics & All patients & Closed reduction & Open reduction \\
\hline Total number & 402 & 201 & 201 \\
\hline Bilateral & $20(5.0 \%)$ & $13(6.5 \%)$ & $7(3.5 \%)$ \\
\hline Age (years) & $75.39(17.78)$ & $75.19(17.70)$ & $75.59(17.93)$ \\
\hline \multicolumn{4}{|l|}{ Gender } \\
\hline Male & $148(36.8 \%)$ & $74(36.8 \%)$ & $74(36.8 \%)$ \\
\hline Female & $254(63.2 \%)$ & $127(63.2 \%)$ & $127(63.2 \%)$ \\
\hline Injury characteristics & All patients & Closed reduction & Open reduction \\
\hline \multicolumn{4}{|l|}{ Mechanism of injury } \\
\hline Low energy & $352(87.6 \%)$ & $176(87.6 \%)$ & $176(87.6 \%)$ \\
\hline High energy & $50(12.4 \%)$ & $25(12.4 \%)$ & $25(12.4 \%)$ \\
\hline Isolated & $347(86.3 \%)$ & $168(83.6 \%)$ & $179(89.1 \%)$ \\
\hline ISS $>16$ & $20(4.9 \%)$ & $11(5.5 \%)$ & $9(4.5 \%)$ \\
\hline \multicolumn{4}{|l|}{ Side } \\
\hline Left & $224(55.7 \%)$ & $117(58.2 \%)$ & $107(53.2 \%)$ \\
\hline Right & $178(44.3 \%)$ & $84(41.8 \%)$ & $94(46.8 \%)$ \\
\hline Open fracture & $4(1.0 \%)$ & $0(0.0 \%)$ & $4(2 \%)$ \\
\hline Medical comorbidities & All patients & Closed reduction & Open reduction \\
\hline \multicolumn{4}{|l|}{ ASA } \\
\hline 1 & $30(7.5 \%)$ & $15(7.5 \%)$ & $15(7.5 \%)$ \\
\hline 2 & $100(24.9 \%)$ & $56(27.9 \%)$ & $44(21.9 \%)$ \\
\hline 3 & $203(50.5 \%)$ & $96(47.8 \%)$ & $107(53.2 \%)$ \\
\hline 4 & $69(17.2 \%)$ & $34(16.9 \%)$ & $35(17.4 \%)$ \\
\hline Charlson comorbidity score & $5.35(2.94)$ & $5.14(2.91)$ & $5.55(2.97)$ \\
\hline Diabetes & $60(14.9 \%)$ & $25(12.4 \%)$ & $35(17.4 \%)$ \\
\hline Steroids & $15(3.7 \%)$ & $8(4.0 \%)$ & $7(3.5 \%)$ \\
\hline Malignancy & $90(22.4 \%)$ & $45(22.4 \%)$ & $45(22.4 \%)$ \\
\hline Dementia & $106(26.4 \%)$ & $49(24.4 \%)$ & $57(28.4 \%)$ \\
\hline Osteoporosis & All patients & Closed reduction & Open reduction \\
\hline Bisphosphonates pre-admission & $70(17.4 \%)$ & $39(19.4 \%)$ & $31(15.4 \%)$ \\
\hline Bisphosphonates on discharge & $100(24.9 \%)$ & $55(28.8 \%)$ & $45(23.9 \%)$ \\
\hline Calcium/vitamin D pre-admission & $127(31.6 \%)$ & $57(28.4 \%)$ & $70(34.8 \%)$ \\
\hline Calcium/vitamin D on discharge & $197(49.0 \%)$ & $90(47.1 \%)$ & $107(56.9 \%)$ \\
\hline Vitamin D loading on admission & $68(16.9 \%)$ & $41(21.5 \%)$ & $27(14.4 \%)$ \\
\hline Fragility fractures before & $99(24.6 \%)$ & $44(21.9 \%)$ & $55(27.5 \%)$ \\
\hline Fragility fractures after & $67(16.7 \%)$ & $42(20.9 \%)$ & $25(12.5 \%)$ \\
\hline \multicolumn{4}{|l|}{ DEXA result } \\
\hline Normal & $3(8.5 \%)$ & $1(4.5 \%)$ & $2(15.4 \%)$ \\
\hline Osteopenia & $10(28.6 \%)$ & $6(27.3 \%)$ & $4(30.8 \%)$ \\
\hline Osteoporosis & $22(62.9 \%)$ & $15(68.2 \%)$ & $7(53.8 \%)$ \\
\hline Social history & All patients & Closed reduction & Open reduction \\
\hline Smoking & $73(18.2 \%)$ & $43(21.4 \%)$ & $30(17.2 \%)$ \\
\hline Alcohol $>10$ units/week & $71(17.7 \%)$ & $37(18.4 \%)$ & $34(16.9 \%)$ \\
\hline \multicolumn{4}{|l|}{ Pre-operative mobility } \\
\hline Independent & $192(47.8 \%)$ & $99(49.3 \%)$ & $93(46.3 \%)$ \\
\hline Stick(s)/crutch(es) & $111(27.6 \%)$ & $52(25.9 \%)$ & $59(29.4 \%)$ \\
\hline Frame & $82(20.4 \%)$ & $40(19.9 \%)$ & $42(20.9 \%)$ \\
\hline Wheelchair/hoisted & $17(4.2 \%)$ & $10(5.0 \%)$ & $7(3.5 \%)$ \\
\hline Frequent falls & $121(30.1 \%)$ & $56(27.9 \%)$ & $65(32.3 \%)$ \\
\hline Operation characteristics & All patients & Closed reduction & Open reduction \\
\hline Operation in less than $48 \mathrm{~h}$ & $319(79.4 \%)$ & $161(80.1 \%)$ & $158(78.6 \%)$ \\
\hline
\end{tabular}


Table 1 (continued)

\begin{tabular}{|c|c|c|c|}
\hline Operation characteristics & All patients & Closed reduction & Open reduction \\
\hline Simultaneous procedures & $24(6.0 \%)$ & $10(5.0 \%)$ & $14(7.0 \%)$ \\
\hline Use of cerclage wires & $50(12.4 \%)$ & $0(0.0 \%)$ & $50(24.9 \%)$ \\
\hline Surgical time (min) & $110.87(44.22)$ & $96.58(35.60)$ & $125.22(47.36)$ \\
\hline Anaesthetic time (min) & $48.14(22.17)$ & $47.97(21.20)$ & $48.30(23.16)$ \\
\hline Time from induction to recovery ( $\mathrm{min}$ ) & $177.70(48.37)$ & $161.65(41.19)$ & $193.82(49.76)$ \\
\hline \multicolumn{4}{|l|}{ Level of first surgeon } \\
\hline Registrar & $244(61.2 \%)$ & $131(65.5 \%)$ & $113(56.8 \%)$ \\
\hline Consultant & $155(38.8 \%)$ & $69(34.5 \%)$ & $86(43.2 \%)$ \\
\hline \multicolumn{4}{|l|}{ Level of senior surgeon present } \\
\hline Registrar & $227(56.9 \%)$ & $123(61.5 \%)$ & $104(52.3 \%)$ \\
\hline Consultant & $172(43.1 \%)$ & $77(38.5 \%)$ & $95(47.7 \%)$ \\
\hline Complications & All patients & Closed reduction & Open reduction \\
\hline Nail related complications* & $81(20.1 \%)$ & $31(15.4 \%)$ & $50(24.9 \%)$ \\
\hline Failure at lag screw junction & $20(5.0 \%)$ & $6(3.0 \%)$ & $14(7.0 \%)$ \\
\hline Self-dynamisation & $20(5.0 \%)$ & $8(4.0 \%)$ & $12(6.0 \%)$ \\
\hline Cut-out & $10(2.5 \%)$ & $5(2.5 \%)$ & $5(2.5 \%)$ \\
\hline Non-union & $63(15.7 \%)$ & $28(13.9 \%)$ & $35(17.4 \%)$ \\
\hline Peri-implant fracture & $12(3.0 \%)$ & $4(2.0 \%)$ & $8(4.0 \%)$ \\
\hline Re-operation (nail failure) ${ }^{* *}$ & $28(7.0 \%)$ & $8(4.0 \%)$ & $20(10 \%)$ \\
\hline Re-operation (any cause)*** & $63(15.7 \%)$ & $28(13.9 \%)$ & $35(17.4 \%)$ \\
\hline HAP/CAP & $83(20.6 \%)$ & $42(20.9 \%)$ & $41(20.4 \%)$ \\
\hline UTI & $63(15.7 \%)$ & $25(12.4 \%)$ & $38(18.9 \%)$ \\
\hline \multicolumn{4}{|l|}{ Wound infection } \\
\hline Superficial & $14(3.5 \%)$ & $2(1.0 \%)$ & $12(6.0 \%)$ \\
\hline Deep & $10(2.5 \%)$ & $2(1.0 \%)$ & $8(4.0 \%)$ \\
\hline \multicolumn{4}{|l|}{ CKD Stage pre-operatively } \\
\hline Mild & $273(69.5 \%)$ & $144(73.5 \%)$ & $129(65.5 \%)$ \\
\hline Moderate/severe & $120(30.5 \%)$ & $52(26.5 \%)$ & $68(34.5 \%)$ \\
\hline \multicolumn{4}{|l|}{ CKD stage post-operatively } \\
\hline Mild & $285(72.7 \%)$ & $156(79.6 \%)$ & $129(65.8 \%)$ \\
\hline Moderate/severe & $107(27.3 \%)$ & $40(20.4 \%)$ & $67(34.2 \%)$ \\
\hline Pre-operative transfusion & $35(8.7 \%)$ & $11(5.5 \%)$ & $24(12.0 \%)$ \\
\hline Post-operative transfusion (48 h) & $212(52.7 \%)$ & $83(41.5 \%)$ & $129(64.5 \%)$ \\
\hline Post-operative transfusion (total) & $258(64.2 \%)$ & $106(53.0 \%)$ & $152(76.0 \%)$ \\
\hline Hb drop $(\mathrm{g} / \mathrm{dL})$ & $45.16(17.92)$ & $48.07(16.70)$ & $42.29(18.65)$ \\
\hline \multicolumn{4}{|l|}{ VTE } \\
\hline No & $78(84.8 \%)$ & $36(85.7 \%)$ & $42(84 \%)$ \\
\hline DVT & $8(8.7 \%)$ & $4(9.5 \%)$ & $4(8 \%)$ \\
\hline PE & $6(6.5 \%)$ & $2(4.8 \%)$ & $4(8 \%)$ \\
\hline Radiographic measurements & All patients & Closed reduction & Open reduction \\
\hline \multicolumn{4}{|l|}{ Femoral neck shaft angle } \\
\hline Normal & $266(67.5 \%)$ & $125(63.5 \%)$ & $141(71.6 \%)$ \\
\hline Coxa Valga & $98(24.9 \%)$ & $55(27.9 \%)$ & $43(21.8 \%)$ \\
\hline Coxa Vara & $30(7.6 \%)$ & $17(8.6 \%)$ & $13(6.6 \%)$ \\
\hline \multicolumn{4}{|l|}{ Number of fragments (comminution) } \\
\hline Simple & $100(24.9 \%)$ & $45(22.4 \%)$ & $55(27.5 \%)$ \\
\hline Moderate & $210(52.4 \%)$ & $115(57.2 \%)$ & $95(47.5 \%)$ \\
\hline Severe & $91(22.7 \%)$ & $41(20.4 \%)$ & $51(24.6 \%)$ \\
\hline Isolated subtrochanteric extension & $61(15.2 \%)$ & $27(13.4 \%)$ & $34(17.0 \%)$ \\
\hline Atypical & $16(3.9 \%)$ & $9(4.5 \%)$ & $7(3.5 \%)$ \\
\hline Pathological & $5(1.2 \%)$ & $4(2.0 \%)$ & $1(0.5 \%)$ \\
\hline
\end{tabular}


Table 1 (continued)

\begin{tabular}{|c|c|c|c|}
\hline Radiographic measurements & All patients & Closed reduction & Open reduction \\
\hline Distal extension & $136(33.8 \%)$ & $49(24.4 \%)$ & $87(43.5 \%)$ \\
\hline Lesser trochanter fracture & $279(69.4 \%)$ & $136(67.7 \%)$ & $143(71.5 \%)$ \\
\hline Medial calcar comminution & $25(6.2 \%)$ & $16(8.0 \%)$ & $9(4.5 \%)$ \\
\hline \multicolumn{4}{|l|}{ Lateral cortex gap size (mm) } \\
\hline$\leq 4$ & $245(61.4 \%)$ & $129(64.8 \%)$ & $116(58.0 \%)$ \\
\hline $5-9$ & $103(25.8 \%)$ & $44(22.1 \%)$ & $59(29.5 \%)$ \\
\hline$\geq 10$ & $51(12.8 \%)$ & $26(13.1 \%)$ & $25(12.5 \%)$ \\
\hline \multicolumn{4}{|l|}{ Medial cortex gap size (mm) } \\
\hline$\leq 4$ & $270(67.7 \%)$ & $141(70.9 \%)$ & $129(64.5 \%)$ \\
\hline $5-9$ & $89(22.3 \%)$ & $39(19.6 \%)$ & $50(25.0 \%)$ \\
\hline$\geq 10$ & $40(10.0 \%)$ & $19(9.5 \%)$ & $21(10.5 \%)$ \\
\hline \multicolumn{4}{|l|}{ Anterior cortex gap size (mm) } \\
\hline$\leq 4$ & $265(66.2 \%)$ & $129(64.5 \%)$ & $136(68.0 \%)$ \\
\hline $5-9$ & $87(21.8 \%)$ & $44(22.0 \%)$ & $43(21.5 \%)$ \\
\hline$\geq 10$ & $48(12.0 \%)$ & $27(13.5 \%)$ & $21(10.5 \%)$ \\
\hline \multicolumn{4}{|l|}{ Posterior cortex gap size $(\mathrm{mm})$} \\
\hline$\leq 4$ & $312(78.0 \%)$ & $161(80.5 \%)$ & $151(75.5 \%)$ \\
\hline $5-9$ & $66(16.5 \%)$ & $28(14.0 \%)$ & $38(19.0 \%)$ \\
\hline$\geq 10$ & $22(5.5 \%)$ & $11(5.5 \%)$ & $11(5.5 \%)$ \\
\hline \multicolumn{4}{|l|}{ Reduction angle grouped (degrees) } \\
\hline Valgus 5 -varus 5 & $290(72.7 \%)$ & $149(74.9 \%)$ & $141(70.5 \%)$ \\
\hline Valgus $>5$ & $29(7.3 \%)$ & $16(8.0 \%)$ & $13(6.5 \%)$ \\
\hline Varus $5-10$ & $5 \mathrm{a} 9(14.8 \%)$ & $25(12.6 \%)$ & $34(17.0 \%)$ \\
\hline Varus $>10$ & $21(5.2 \%)$ & $9(4.5 \%)$ & $12(6.0 \%)$ \\
\hline \multicolumn{4}{|l|}{$\mathrm{TAD}(\mathrm{mm})$} \\
\hline$<25$ & $353(89.6 \%)$ & $175(89.3 \%)$ & $178(89.9 \%)$ \\
\hline$\geq 25$ & $41(10.4 \%)$ & $21(10.7 \%)$ & $20(10.1 \%)$ \\
\hline Distal locking 1 & $10(2.5 \%)$ & $7(3.5 \%)$ & $3(1.5 \%)$ \\
\hline (Number of screws) 2 & $391(97.5 \%)$ & $194(96.5 \%)$ & $197(98.5 \%)$ \\
\hline \multicolumn{4}{|l|}{ Method of locking } \\
\hline Static Locking & $263(66.1 \%)$ & $132(66.0 \%)$ & $131(66.2 \%)$ \\
\hline Secondary dynamisation & $133(33.4 \%)$ & $67(33.5 \%)$ & $66(33.3 \%)$ \\
\hline Dynamic & $2(0.5 \%)$ & $1(0.5 \%)$ & $1(0.5 \%)$ \\
\hline Nail/canal ratio & $0.82(0.08)$ & $0.82(0.08)$ & $0.82(0.08)$ \\
\hline Hospital stay/mortality & All patients & Closed reduction & Open reduction \\
\hline HDU/ICU stay & $48(11.9 \%)$ & $21(10.4 \%)$ & $27(13.4 \%)$ \\
\hline Total length of hospital stay (days) & $23.71(19.25)$ & $23.01(19.80)$ & $24.42(18.70)$ \\
\hline Weekend admission & $134(33.3 \%)$ & $68(33.8 \%)$ & $66(32.8 \%)$ \\
\hline Died within a year & $72(17.9 \%)$ & $29(14.4 \%)$ & $43(21.4 \%)$ \\
\hline
\end{tabular}

Dichotomous variables are presented as absolute numbers (percentages) of the positive event

Continuous variables are presented as mean (SD)

ISS injury severity score; $A S A$ American society of anaesthesiologists classification; $D E X A$ dual-energy X-ray absorptiometry; $F W B$ full weight bearing; $P W B$ partial weight bearing; $T T W B$ toe-touch weight bearing; $N W B$ non-weight bearing; $H A P$ hospital acquired pneumonia; $C A P$ community acquired pneumonia; UTI urinary tract infection; $C K D$ chronic kidney disease; $D V T$ deep vein thrombosis; VTE venous thromboembolism; TAD tip apex distance; $A P$ anterior-posterior view; $L A T$ lateral view; $H D U$ high dependency unit; $I C U$ intensive care unit

*Nail related complications: this included nail failure, peri-implant fracture and peri-implant infection

**Nail failure: this included failure at lag screw junction, cut-out of the lag screw and breakage of the distal locking screws (self-dynamisation) as nail-failure

${ }^{* * * *}$ Re-operation for all causes included re-operation following nail failure, infection, removal of metalwork for any cause (i.e. impingement, posttraumatic arthritis, removal of distal screws for dynamisation of the nail) and revision for non-union 
Table 2 Unadjusted associations with open reduction

\begin{tabular}{lll}
\hline Operation characteristics & Unadjusted OR (95\% CI) & $p$ value \\
\hline Surgical time (>120 min) & $3.31(2.15-5.10)$ & $<0.001$ \\
\hline Complications & $\begin{array}{l}\text { Unadjusted OR } \\
(95 \% \text { CI })\end{array}$ & 0.019 \\
\hline Nail related complications & $1.82(1.10-2.99)$ & \\
Wound infection & $6.53(1.44-29.58)$ & 0.014 \\
Superficial & $4.35(0.91-20.77)$ & 0.065 \\
Deep & $2.56(1.71-3.84)$ & $<0.001$ \\
Post-operative transfusion & $2.81(1.83-4.30)$ & $<0.001$ \\
(48 h) & $0.98(0.97-0.99)$ & 0.002 \\
Post-operative transfusion (total) & $\begin{array}{l}\text { Unadjusted OR } \\
\text { Hb drop (g/dL) }\end{array}$ & $(95 \%$ CI $)$ \\
\hline Radiographic measurements & $2.39(1.56-3.66)$ & $<0.001$ \\
\hline Distal extension & & \\
\hline
\end{tabular}

Table 3 Outcomes of unmatched patients presenting with a deep infection

\begin{tabular}{llll}
\hline & Closed reduction & Open reduction & \\
\cline { 3 - 4 } \cline { 3 - 3 } & & No cerclage wiring & Cerclage wiring \\
\hline Number of pts & $3 \mathrm{pts}$ & $7 \mathrm{pts}$ & $3 \mathrm{pts}$ \\
Re-operations & $2 \mathrm{pts}$ & $6 \mathrm{pts}$ & $2 \mathrm{pts}$ \\
Number of re-operations & One pt had 2 and & Mean: 6 re-operations & One pt had 1 and \\
& another 5 re-opera- & Median: 6 re-operations & another 3 re- \\
Final outcome (union) & tions & SD: 4.2 re-operations & operations \\
Infection outcome & Remission: 3 pts & Ron-union: 3 pts** & Non-union: 1 pt**** \\
Mortality & $1 \mathrm{pt}$ & 3 pts & Remission: 3 pts \\
\hline
\end{tabular}

pt patient

*One patient died before union and one patient had a total hip replacement secondary to non-union

**Two patients died before union; one patient had a total hip replacement secondary to non-union; one patient had an asymptomatic infected non-union and managed conservatively

***In one patient the infection lead to multi-organ failure resulting in patient's death

*****Patient died before union

\section{Discussion}

Subtrochanteric fractures have been previously associated with a high risk of complications, some of which could be

Table 4 Multivariate models demonstrating associations of open reduction following a subtrochanteric fracture

\begin{tabular}{lllr}
\hline & OR & $95 \%$ CI & $\operatorname{Pr}(>|\mathrm{z}|)$ \\
\hline Wound infection (superficial) & 7.88 & $1.63-38.16$ & 0.010 \\
Wound infection (deep) & 3.21 & $0.63-16.33$ & 0.160 \\
Surgical time (>120 min) & 3.09 & $1.96-4.87$ & $<0.001$ \\
$\begin{array}{l}\text { Transfusion within 48 h post-opera- } \\
\quad \text { tively }\end{array}$ & 2.44 & $1.58-3.76$ & $<0.001$ \\
Distal extension & 2.30 & $1.45-3.65$ & $<0.001$ \\
\hline
\end{tabular}

$O R$ odds ratio, $C I$ confidence interval
Table 5 Multivariate models demonstrating associations of 'clamp assisted only' open reduction vs. open reduction using cerclage wire/ cable following a subtrochanteric fracture

\begin{tabular}{lrlr}
\hline & \multicolumn{1}{c}{ OR } & $95 \%$ CI & $\operatorname{Pr}(>|z|)$ \\
\hline Distal extension & 10.23 & $4.81-21.78$ & $<0.001$ \\
Surgical time $>120 \mathrm{~min}$ & 6.43 & $3.12-13.26$ & $<0.001$ \\
Non-union & 0.20 & $0.06-0.74$ & 0.015 \\
\hline
\end{tabular}

$O R$ odds ratio, $C I$ confidence interval 
potentially preventable [22-24]. In the literature, there is still a lack of evidence comparing the outcomes of open versus closed reduction [22, 25-28]. Most of these studies are small in sample size [22, 25, 27], whilst some assess the elderly age group only [27], but no study reports on the associations of open reduction. Our study, therefore, represents the first study reporting on the associations of open reduction in subtrochanteric femur fractures and related complications.

Open reduction has traditionally been met with some reluctance. This is due to the misbelief that open reduction may compromise the local fracture biology and vascularity [5], thereby reducing the threshold of what could be deemed as an 'acceptable' suboptimal reduction. Similarly, cerclage wiring was believed to cause strangulation of the bone, risking necrosis and ultimately failure of healing [29]. Recent studies have, however, challenged this school of thought. Histological and anatomical studies by Nather et al. [30] and Pazzaglia et al. [31] have both described the periosteal blood supply as circumferential. Lenz et al.'s ex-vivo study using human diaphyseal bone further confirmed this, whereby they found no periosteal compression when using a cerclage wire or a cable $[32,33]$. Additionally, in a systematic review by Förch et al. on the effect on cerclage wiring to periosteal perfusion, the group reported no significant evidence to support this [34]. Furthermore, biomechanical studies have also demonstrated how the use of cerclage wire is advantageous, as it stabilises the medial hinge and raises the threshold of cyclical compressive loading tolerated by the femur before reaching plastic deformation [35] — factors found to significantly decrease the rate of implant failure following IM nailing [35].

Contrary to the bimodal age and gender distribution [2], and unimodal older male and female distribution reported in the literature [36-38], we identified a bimodal male, unimodal older female age distribution in our cohort. The majority of subtrochanteric fractures (82.4\%) in our adult population had comminution or distal diaphyseal extension-all characteristics of an unstable fracture pattern. This, therefore, explains the high rate of open reduction demonstrated in our study population (48\%), which is in keeping with that reported by other studies (range: 33.3-82.2\%) [25-27]. To reduce the bias of our outcomes, we matched the two groups as per age, gender and mechanism of injury. Only distal fracture extension to the diaphysis was found to be different $(p<0.001)$, whilst the rest of the fracture characteristics were comparable in the two groups. Furthermore, our regression analysis confirmed that open reduction was associated with a longer surgical time (OR: 3.09), which suggests a more challenging fracture reduction mandating open reduction in the first place.

Kilinc et al. reported a 5.8\% superficial and 3.9\% of deep infection in their study on 52 patients with cerclage wiring of subtrochanteric fractures treated with IM nailing [39].
Studies comparing open and closed reduction of subtrochanteric fractures treated with cephalomedullary nailing reported similar infection rates between the two groups, which range between 0 and $1.7 \%$ [25-28, 40]. These studies, however, are limited by their small sample size and restricted age groups. Having overcome the aforementioned limitations, our study has conclusively revealed that there is a statistically significant higher risk of developing superficial wound infection in the open reduction group (OR: 7.88). Contrary to common belief, deep infection was not statistically increased amongst the open reduction group.

There is a paucity of studies in the literature which compared the blood transfusion requirement between subtrochanteric fractures requiring open versus closed reduction. Codesido et al. reported no difference in transfusion requirements between the two groups [26], whereas Shukla et al. reported higher transfusion requirement in the open reduction group [27]. We found that open reduction poses a higher risk of requiring blood transfusion within the initial 48 -h postoperative period (OR: 2.44). The higher transfusion risk could be explained by our findings wherein open reduction was performed in fractures requiring a prolonged surgical time, more extensive surgical dissection and, therefore, soft tissue damage-all contributory factors towards a higher bleeding risk.

Collectively, we found that open reduction was associated with a higher risk of nail complications than closed reduction $(p=0.031)$. However, this difference was no longer significant when we individually assessed each of the nail complications. The small sample size for each of these complications and, therefore, an underpowered comparison (Type II error) most likely accounts for the lack of statistical difference.

With regard to the non-union rates, these have been reported to be lower in open reduction group [25, 26]. Karayiannis et al. reported rates of symptomatic non-union to be $5.8 \%$ and $6.3 \%$ in the open reduction and closed reduction group, respectively [25]. Codesido reported no nonunions amongst subtrochanteric fractures requiring open reduction, whilst $8.3 \%$ of the closed reduction group developed non-union [26]. Kilinc et al. (cerclage wire reduction) and Mingo-Robinet et al. (clamp assisted reduction only) both reported no non-unions in their cohorts of patients $[28,39]$. Out of the three main operative factors, Krappinger et al. found that the lack of medial cortical support and varus malalignment as significant risk factors for nonunion in subtrochanteric fractures treated by IM nailing [3]. Even though our study has demonstrated no difference in the non-union rates between subtrochanteric fractures treated with closed or open reduction, we did identify that open anatomical reduction achieved by cerclage wire/cable to have a lower non-union rate (OR: 0.20), when compared to 'clamp assisted only' open reduction. One could assume that 
higher non-union rates seen in open reduction group were secondary to suboptimal exposure and reduction. Interestingly, the use of cerclage wires/cables seems to reduce the risk of non-union. Possible mechanisms behind this could be a better reduction of the fracture with smaller fracture gaps and restoration of the medial buttress.

To our knowledge, our study is the largest cohort series reported in the literature to date comparing the outcomes of closed versus open reduction of subtrochanteric femur fractures treated with IM nailing. With no exclusion criteria posed upon age or comorbidity, our study provides a better overview of the overall fracture demographics and epidemiology of subtrochanteric femur fractures encountered in a Level 1 Trauma Centre serving a metropolitan population. By matching the two groups with respect to age, gender and mechanism of injury, we reduced bias that could result from baseline differences between the two populations. The retrospective nature of our study on the other hand meant certain data may be subjected to bias. Although redness, swelling and mild discharge in the first few post-operative days are signs of physiological inflammation of wound healing, this could easily be clinically over-diagnosed as 'superficial wound infection' by non-orthopaedic physicians, junior doctors, district nurses or advance nursing practitioners who are less experienced in assessing surgical wounds. Therefore, we may have overestimated this risk, with an inherent bias of superficial infection towards open reduction. We also accept that the assessment of fracture pattern is subject to intra- and inter-observer reliability. We did, however, attempt to overcome this weakness by having two independent assessors for the analysis of fracture pattern and radiological measurements. Of note, fractures requiring an open reduction are generally more difficult to reduce and, therefore, are subjected to a higher risk of complications including non-union, hence a potential bias against this group.

\section{Conclusion}

Open reduction of subtrochanteric fractures is not associated with an increased risk of deep infection and non-union. It is, however, associated with an increased risk of superficial infection, transfusion within $48 \mathrm{~h}$ following surgery and a prolonged surgical time. Furthermore, we found that open anatomical reduction achieved by cerclage wiring to have a lower non-union rate when compared to "clamp assisted only' open reduction. Optimal anatomical fracture reduction of subtrochanteric femur fractures during primary surgery should, therefore, be prioritised, even if an open reduction is required. We do not, however, advocate open reduction of fractures where adequate reduction can be achieved by closed means, as this could be linked to an unnecessary risk of complications.
Funding No funding was received for the completion of this project. All work was performed at Leeds General Infirmary and University of Leeds.

Data availability The data that support the findings of this study are available from the corresponding author, MP, upon reasonable request.

\section{Declarations}

Conflict of interest None of the authors has any conflict of interest relevant top this study.

Ethical approval This study was conducted with IRB approval from the Leeds Teaching Hospitals (LTH\#2591).

Open Access This article is licensed under a Creative Commons Attribution 4.0 International License, which permits use, sharing, adaptation, distribution and reproduction in any medium or format, as long as you give appropriate credit to the original author(s) and the source, provide a link to the Creative Commons licence, and indicate if changes were made. The images or other third party material in this article are included in the article's Creative Commons licence, unless indicated otherwise in a credit line to the material. If material is not included in the article's Creative Commons licence and your intended use is not permitted by statutory regulation or exceeds the permitted use, you will need to obtain permission directly from the copyright holder. To view a copy of this licence, visit http://creativecommons.org/licenses/by/4.0/.

\section{References}

1. Wiss DA, Brien WW. Subtrochanteric fractures of the femur. Results of treatment by interlocking nailing. Clin Orthop Relat Res. 1992;1992:231-6.

2. Joglekar SB, Lindvall EM, Martirosian A. Contemporary management of subtrochanteric fractures. Orthop Clin North Am. 2015;46:21-35.

3. Krappinger D, Wolf B, Dammerer D, Thaler M, Schwendinger P, Lindtner RA. Risk factors for nonunion after intramedullary nailing of subtrochanteric femoral fractures. Arch Orthop Trauma Surg. 2019;139:769-77.

4. Russell TATJ. Subtrochanteric fractures of the femur. Philadelphia: WB Saunders; 1992. p. 1485-524.

5. Panteli M, Mauffrey C, Giannoudis PV. Subtrochanteric fractures: issues and challenges. Injury. 2017;48:2023-6.

6. Loizou CL, McNamara I, Ahmed K, Pryor GA, Parker MJ. Classification of subtrochanteric femoral fractures. Injury. 2010;41:739-45.

7. Koch JC. The laws of bone architecture. Am J Anat. 1917;21:177-298.

8. GJ. Haidukewych JL (2010) Subtrochanteric fractures Rockwood and Green's fractures in adults: Lippincott Williams \& Wilkins, Philadelphia, p 1641-1654

9. Santolini E, Goumenos SD, Giannoudi M, Sanguineti F, Stella M, Giannoudis PV. Femoral and tibial blood supply: a trigger for non-union? Injury. 2014;45:1665-73.

10. Matre K, Havelin LI, Gjertsen JE, Vinje T, Espehaug B, Fevang JM. Sliding hip screw versus IM nail in reverse oblique trochanteric and subtrochanteric fractures. A study of 2716 patients in the Norwegian hip fracture register. Injury. 2013;44:735-42.

11. Kim JW, Park KC, Oh JK, Oh CW, Yoon YC, Chang HW. Percutaneous cerclage wiring followed by intramedullary nailing for 
subtrochanteric femoral fractures: a technical note with clinical results. Arch Orthop Trauma Surg. 2014;134:1227-35.

12. Apivatthakakul T, Phaliphot J, Leuvitoonvechkit S. Percutaneous cerclage wiring, does it disrupt femoral blood supply? A cadaveric injection study. Injury. 2013;44:168-74.

13. Persiani P, Noia G, de Cristo C, Graci J, Gurzi MD, Villani C. A study of 44 patients with subtrochanteric fractures treated using long nail and cerclage cables. Musculoskelet Surg. 2015;99:225-30.

14. Malik MH, Harwood P, Diggle P, Khan SA. Factors affecting rates of infection and nonunion in intramedullary nailing. $\mathrm{J}$ Bone Jt Surg Br Vol. 2004;86:556-60.

15. Tang P, Gates C, Hawes J, Vogt M, Prayson MJ. Does open reduction increase the chance of infection during intramedullary nailing of closed tibial shaft fractures? J Orthop Trauma. 2006;20:317-22.

16. Imerci A, Aydogan NH, Tosun $\mathrm{K}$. Evaluation of inter- and intraobserver reliability of current classification systems for subtrochanteric femoral fractures. Eur J Orthop Surg Traumatol. 2018;28:499-502.

17. Müller ME, Nazarian S, Koch P, Schatzker J. The comprehensive classification of fractures of long bones. Berlin: Springer Science \& Business Media; 2012.

18. Raff AB, Kroshinsky D. Cellulitis: a review. JAMA. 2016;316:325-37.

19. Ban KA, Minei JP, Laronga C, Harbrecht BG, Jensen EH, Fry DE, et al. American College of Surgeons and Surgical Infection Society: surgical site infection guidelines, 2016 update. J Am Coll Surg. 2017;224:59-74.

20. R Foundation for Statistical Computing V, Austria R: A language and environment for statistical computing.

21. Lewer D. Exact Matching in R. 2020.

22. Hoskins W, Bingham R, Joseph S, Liew D, Love D, Bucknill A, et al. Subtrochanteric fracture: the effect of cerclage wire on fracture reduction and outcome. Injury. 2015;46:1992-5.

23. Giannoudis PV, Ahmad MA, Mineo GV, Tosounidis TI, Calori GM, Kanakaris NK. Subtrochanteric fracture non-unions with implant failure managed with the "Diamond" concept. Injury. 2013;44(Suppl 1):S76-81.

24. Park SH, Kong GM, Ha BH, Park JH, Kim KH. Nonunion of subtrochanteric fractures: comminution or malreduction. Pak J Med Sci. 2016;32:591-4.

25. Karayiannis P, James A. The impact of cerclage cabling on unstable intertrochanteric and subtrochanteric femoral fractures: a retrospective review of 465 patients. Eur J Trauma Emerg Surg. 2019;46(5):969-975. https://doi.org/10.1007/ s00068-018-01071-4

26. Codesido P, Mejia A, Riego J, Ojeda-Thies C. Subtrochanteric fractures in elderly people treated with intramedullary fixation: quality of life and complications following open reduction and cerclage wiring versus closed reduction. Arch Orthop Trauma Surg. 2017;137:1077-85.
27. Shukla S, Johnston P, Ahmad MA, Wynn-Jones H, Patel AD, Walton NP. Outcome of traumatic subtrochanteric femoral fractures fixed using cephalo-medullary nails. Injury. 2007;38:1286-93.

28. Mingo-Robinet J, Torres-Torres M, Moreno-Barrero M, Alonso JA, Garcia-Gonzalez S. Minimally invasive clamp-assisted reduction and cephalomedullary nailing without cerclage cables for subtrochanteric femur fractures in the elderly: surgical technique and results. Injury. 2015;46:1036-41.

29. Perren SM, Fernandez Dell'Oca A, Lenz M, Windolf M. Cerclage, evolution and potential of a Cinderella technology. An overview with reference to periprosthetic fractures. Acta Chir Orthop Traumatol Cech. 2011;78:190-9.

30. Nather A, Ong H, Aziz Z (2005) Structure of bone. bone grafts and bone substitutes: basic science and clinical applications. World Scientific Publishing Company; 2005. p 3-17.

31. Pazzaglia UE, Congiu T, Raspanti M, Ranchetti F, Quacci D. Anatomy of the intracortical canal system: scanning electron microscopy study in rabbit femur. Clin Orthop Relat Res. 2009;467:2446-56.

32. Lenz M, Perren SM, Gueorguiev B, Richards RG, Krause F, Fernandez Dell'Oca A, et al. Underneath the cerclage: an ex vivo study on the cerclage-bone interface mechanics. Arch Orthop Trauma Surg. 2012;132:1467-72.

33. Lenz M, Perren SM, Richards RG, Muckley T, Hofmann GO, Gueorguiev B, et al. Biomechanical performance of different cable and wire cerclage configurations. Int Orthop. 2013;37:125-30.

34. Forch S, Sandriesser S, Fenwick A, Mayr E. Impairment of the blood supply by cerclages: myth or reality?: An overview of the experimental study situation. Unfallchirurg. 2021;124:231-40.

35. Muller T, Topp T, Kuhne CA, Gebhart G, Ruchholtz S, Zettl R. The benefit of wire cerclage stabilisation of the medial hinge in intramedullary nailing for the treatment of subtrochanteric femoral fractures: a biomechanical study. Int Orthop. 2011;35:1237-43.

36. Yoon BH, Lee YK, Kim SC, Kim SH, Ha YC, Koo KH. Epidemiology of proximal femoral fractures in South Korea. Arch Osteoporos. 2013;8:157.

37. Mattisson L, Bojan A, Enocson A. Epidemiology, treatment and mortality of trochanteric and subtrochanteric hip fractures: data from the Swedish fracture register. BMC Musculoskelet Disord. 2018;19:369.

38. Howard A, Giannoudis PV. Proximal femoral fractures: issues and challenges. Injury. 2012;43:1975-7.

39. Kilinc BE, Oc Y, Kara A, Erturer RE. The effect of the cerclage wire in the treatment of subtrochanteric femur fracture with the long proximal femoral nail: a review of 52 cases. Int J Surg (London, England). 2018;56:250-5.

40. Trikha V, Das S, Agrawal P, Arkesh M, Kumar Dhaka S. Role of percutaneous cerclage wire in the management of subtrochanteric fractures treated with intramedullary nails. Chin J Traumatol. 2018;21:42-9. 